\title{
Animal Models of Parkinson's Disease: New models provide greater translational and predictive value
}

Kevin M. Gamber, PhD

Director, Marketing and Business Development, Horizon Discovery

\section{Overview}

Parkinson's disease (PD) is a progressive neurodegenerative condition that results in movement and cognitive disorders. PD arises from the degeneration of dopamine (DA) neurons in the substantia nigra. Loss of striatal dopaminergic function in PD leads to resting tremor, bradykinesia, rigidity, and postural instability.

The cause of PD is unknown. Most cases are sporadic, however $15 \%$ of patients have a first degree relative with the disease (Samii A, 2004) and up to 5\% of cases have been linked to genes known to be associated with PD (Lesage S, 2009). Treatment measures have been mostly focused on augmentation of dopaminergic signaling: pharmacologic treatments include levodopa (a dopamine precursor), dopamine agonists, and MAO-B inhibitors (prevent dopamine metabolism). These drugs are more focused on symptomatic rather than disease modifying treatment; there is great necessity for large research efforts to develop disease modifying agents that slow progression or even prevent or reverse PD (Savitt JM, 2006).

Animal models are a key part of preclinical research in drug discovery. Chemically-induced models of PD are most often rats administered chemicals toxic to dopaminergic neurons. Genetic models of PD have focused on mutating or knocking out genes known to cause familial PD. As discussed below, rodent models have thus far faced limitations due to lack of strong construct and/or face validity, as well as species and strain limitations.

\section{Chemically-induced models}

Two of the most widely used animal models of Parkinson's disease are the chemically induced 1-methyl-4-phenyl-1,2,3,6-tetrahydropyridine (MPTP) and 6-hydroxydopamine (6-OHDA) rat. Both MPTP and 6-OHDA are neurotoxic and are substrates for the dopamine transporter (DAT). 6-OHDA can also be a substrate for the norepinephrine transporter (NET), and thus, when used alone, 6-OHDA is not solely specific for DA neurons. Both MPTP and 6-OHDA are typically used in rats rather than mice, due to the larger size (easier surgeries and microinjections), richer behavioral profile, and greater translational relevance.

The chemically-induced models of PD have strong face validity for both the loss of dopaminergic neurons and the elicited motor phenotype. They are useful models of dopaminergic loss, especially where the cause of the loss is not important for the study. However, these models have particularly poor construct validity in that they only represent loss of dopaminergic neurons and cannot be used to investigate progression of the pathology of the disease, including the mechanisms responsible for neurodegeneration. Additionally, these models do not form lewy bodies, an important hallmark of PD, and they cannot be used to assess any of the non-dopaminergic changes known to be present in $\mathrm{PD}$.

\section{Genetic models}

Genetic models of PD have focused on genes known to be implicated in familial Parkinson's disease. In these models, the gene of interest is usually either knocked out, or a humanized version of the gene (incorporating a known human mutation) is either knocked in or transgenically expressed (Dawson TM, 2010). Historically, these models have been in mouse due to the ease of genetic manipulation in this species. Target genes in genetic models typically include alpha synuclein (Snca), leucine rich repeat kinase 2 (Lrrk2), parkin (Park2), PTEN-induced putative kinase 1 (Pink1), and DJ-1 (Park7).

The genetic models offer much higher construct validity than the chemically-induced models-at least for individuals with familial PD har- 
boring mutations in these particular genes. For sporadic PD, the construct validity is less clear. While sporadic patients by definition do not possess mutations in the above genes, the underlying pathology and molecular mechanisms are likely to converge at some point. Further, while the context may not be identical, the genetic models, unlike the chemicallyinduced models, do offer the possibility to directly examine PD progression and pathology.

While the genetic models excel in construct validity, they have thus far been disappointing when it comes to face validity. Phenotypes seen in the existing mouse models have been mild at best, with some models exhibiting no real phenotype (Dawson TM, 2010). An ideal model should display a progressive loss of dopamine neurons and formation of lewy bodies, which is then manifested as motor deficits. Non-dopaminergic aspects of PD should also be modeled. To date, no genetic mouse model fulfills these criteria (Dawson TM, 2010).

As mentioned, traditional genetic models of $\mathrm{PD}$ have been made in mouse. Until very recently, mouse has been the only real mammalian option for genetic manipulation. Compared to the rat, the mouse is smaller, exhibits a simpler yet more erratic behavioral profile, and on the whole is less predictive and translational. The advent of zinc finger nucleases (ZFNs), however, enables genetic engineering in animals other than mouse, including the more predictable rat (Geurts AM, 2009). SAGE Labs, part of Horizon Discovery Group plc has harnessed the species flexibility of ZFNs to produce knockout rat models of PD. Developed in collaboration with the Michael J. Fox Foundation, SAGE Labs has commercialized knockout rats for Lrrk2, Parkin, Pink1, and DJ-1.

Unlike the genetic mouse models, early work on knockout rat PD models has been encouraging. Pink1 and DJ-1 knockout rats display a progressive loss of dopaminergic neurons in the substantia nigra, with a 50\% loss by 8 months of age (figure 1). This is the first observation of dopamine cell loss in any genetic model of PD. Moderate to severe motor deficits are coupled with the loss of dopaminergic neurons. Specifically, the Pink1 and DJ-1 knockout rats show impaired performance on the tapered balance beam and hind limb fatigue assays, as well as gait abnormalities as
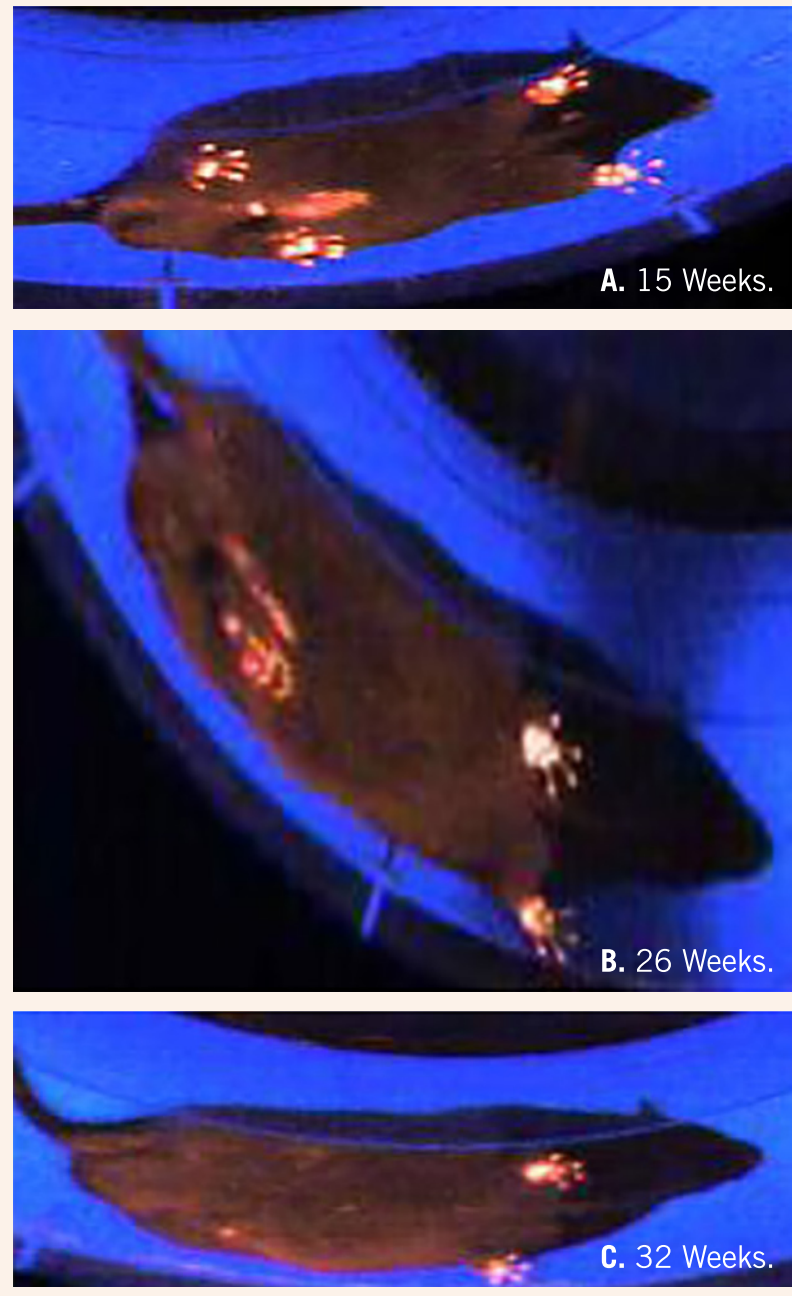

Figure 2. Progressive motor dysfunction in Pink1 knockout rats. Pink1 rats show progressive motor deficits in the hind limbs as assessed by NeuroCube at 15 (a), 26 (b), and 32 (c) weeks of age. assessed by NeuroCube (figure 2). A subpopulation ( 30\%) of Pink1 and DJ-1 knockout rats also show a marked, severe dragging of the hind limbs with onset at around 5 months.

Initial results have demonstrated that the Pink1 knockout rats also possess non-motor and non-dopaminergic characteristics of PD. Anosmia, or the loss of the sense of smell, is frequently reported as an early symptom in human PD patients. Awake Pink1 knockout rats displayed deficits in olfactory circuits as assessed by fMRI. The fMRI also revealed lack of activation of downstream centers, suggesting anhedonia, another symptom frequently seen in PD. Diffusion tensor imaging (DTI) in Pink1 KO rats further revealed altered DTI signals not only in the expected dopaminergic regions, but in non-dopaminergic regions as well. The rat is particularly well suited for imaging studies such as these, as its increased size offers higher resolution as compared to mouse.

In all, the PD rat models from Horizon Discovery's SAGE Labs offer exciting new tools for PD researchers. They allow study in the more preferable and translational rat, and exhibit PD phenotypes not seen in the mouse. These models allow for unprecedented access to study PD-relevant neurodegeneration of dopaminergic neurons in an intact animal. Additionally, unlike chemically-induced models, they allow for the study of PD progression and pathology, an aspect that is absolutely critical to develop therapeutics that can be used to halt disease progression or even prevent PD outright.

\section{References}

Dawson TM, K. H. (2010). Genetic Animal Models of Parkinson's Disease. Neuron, 646-661.

Geurts AM, C. G. (2009). Knockout rats via embryo microinjection of zinc-finger nucleases. Science, 433.

Lesage S, B. A. (2009). Parkinson's disease: from monogenic forms to genetic susceptibility factors. Hum. Mol. Genet, R48-49.

Samii A, N. J. (2004). Parkinson's disease. Lancet, 1783-93.

Savitt JM, D. V. (2006). Diagnosis and treatment of Parkinson disease: molecules to medicine. J Clin Invest, 116: 1744-1754. 